\title{
Microstrip Wideband Diplexer with Narrow Guard Band Based on All-Resonator Structures
}

\author{
Yun Wu, Yi Wang, Eugene A. Ogbodo \\ Engineering Science \\ University of Greenwich (Medway campus), \\ Chatham Maritime, Kent, U.K. \\ y.wu@gre.ac.uk; yi.wang@gre.ac.uk
}

\begin{abstract}
This paper presents a wideband microstrip diplexer with adjacent channels based on an all-resonator structure. The diplexer demonstrates an overall bandwidth of $23.7 \%$ between $1.71 \mathrm{GHz}$ and $2.17 \mathrm{GHz}$ and a narrow guard band of only $40 \mathrm{MHz}$. Two trisection resonator structures are introduced to generate asymmetric transmission zeros to increase isolation between the two channels. The coupling matrix for the all-resonator diplexer is acquired. The diplexer is designed and fabricated on a Rogers RO4003C substrate. The measured results show insertion losses of $1.31 \mathrm{~dB}$ and $1.2 \mathrm{~dB}$ in the two channels, and an isolation of over $28 \mathrm{~dB}$. These results are in good agreement with the simulation.
\end{abstract}

Keywords-diplexer; adjacent channels; cascaded trisection; all resonator structures

\section{INTRODUCTION}

Diplexers and multiplexers are widely used for sharing a single antenna between a transmitter and a receiver or between multiple channels. They are key passive components in many modern wireless communication systems such as mobile communication systems employing frequency division duplex (FDD) [1]. Huge efforts have been made on diplexers and multiplexers with different structures [2]-[7]. Among these, planar structures are particularly popular because of its ease of fabrication, compact size and low-cost integration. The conventional approach of designing a diplexer is to optimize a transmission-line based T-junction or Y-Junction to connect two separately designed channel filters. In recent years, a new approach based on resonant junctions has been developed to replace the transmission-line junctions in search of a more compact multi-port topology. Lots of work has been focused on this type of structures and several synthesis and optimization methods have been developed [8]-[15]. Using this new approach, channel filters are connected via resonator junctions. The diplexer can be characterized by a single coupling matrix and the interaction between channel filters can be taken into account during the coupling matrix synthesis. However, due to the resonant nature of the junction and bandwidth limitation of physical resonators, the capability of such a diplexer topology to achieve a wide overall bandwidth has been a concern [15]. It is particularly challenging when the guard band is narrow because interaction between the two channel filters increases as the guard band decreases. This problem could be further exacerbated in microstrip diplexers because of the limited range of the achievable electromagnetic coupling from the microstrip structures.

This work aims to tackle these challenges and demonstrate a wideband diplexer with a narrow guard band using the microstrip technology. The diplexer is designed based on an all-resonator structure and one common resonator is shared by both channel filters [14]. To achieve the required isolation between the two channels, a trisection-filter structure [16] is introduced in each channel so that a transmission zero can be generated in the adjacent channel.

\section{DIPLEXER DESIGN}

\section{A. Topology and coupling matrix}

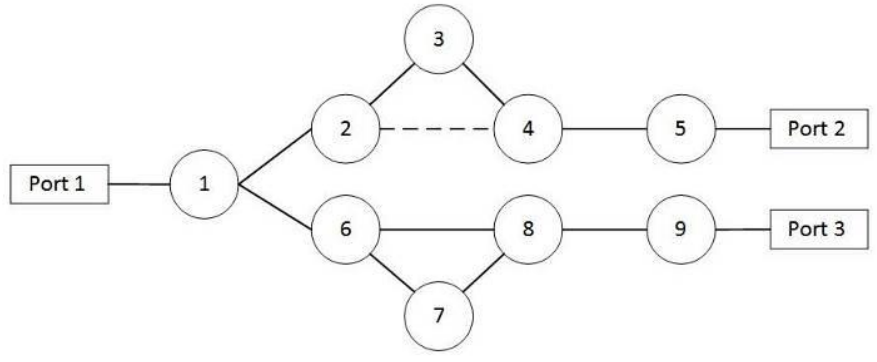

Fig. 1. Topology of the proposed diplexer.

The frequency ranges for the two channels of the microstrip diplexer are 1.71-1.88 GHz (channel A) and 1.92-2.17 GHz (channel B), respectively. The guard band between the two channels is $40 \mathrm{MHz}$, only $8.7 \%$ of the overall bandwidth. The topology of the proposed diplexer is shown in Fig. 1. Each circle denotes a resonator. The lines between them denote the electromagnetic coupling, where the dashed line denotes the opposite sign of the coupling. Signal paths 1-2-3-4-5 and 1-67-8-9 correspond to the channel $\mathrm{A}$ and the channel $\mathrm{B}$, respectively. Resonator 1 is the common resonator shared by the two channels. To increase isolation between the two channels, two trisection structures 2-3-4 and 6-7-8 are introduced in the two channels respectively. The opposite sign 
of the cross couplings between 2 and 4 and between 6 and 8 generates two transmission zeros at the high side $(1.92 \mathrm{GHz})$ of channel $\mathrm{A}$ and low side $(1.88 \mathrm{GHz})$ of channel $\mathrm{B}$.

Based on the topology in Fig. 1, a coupling matrix is acquired. Generally, a normalized matrix [A] can be written as [17]

$$
[A]=[q]+p[U]-j[m]
$$

where $[U]$ is the $N \times N$ identity matrix, $[\mathrm{m}]$ is the normalized coupling matrix. $[q]$ is a diagonal matrix with only $[q]_{i i}=1 / q_{e, i}$ $\neq 0, q_{e, i}$ is the normalized external quality factor from the port attached to the $i$-th resonator and

$$
p=j \cdot\left(f / f_{0}-f_{0} / f\right) / F B W
$$

where $F B W$ is the fractional bandwidth and $f_{0}$ is the central frequency of the overall bandwidth of the diplexer. In this design,

$$
F B W=\left(f_{B, 2}-f_{A, 1}\right) / f_{0}, f_{0}=\sqrt{f_{A, 1} \cdot f_{B, 2}}
$$

where $f_{\mathrm{A}, 1}=1.71 \mathrm{GHz}, f_{\mathrm{A}, 2}=1.88 \mathrm{GHz}, f_{\mathrm{B}, 1}=1.92 \mathrm{GHz}$ and $f_{\mathrm{B}, 2}$ $=2.17 \mathrm{GHz}$. The S-parameter of a multi-port network can be described as

$$
\begin{gathered}
S_{n n}= \pm\left(1-\frac{2}{q_{e, n}}[A]_{n n}^{-1}\right) \\
\left.S_{m n}\right|_{m \neq n}=\frac{2}{\sqrt{q_{e, n} \cdot q_{e, m}}}[A]_{m n}^{-1}
\end{gathered}
$$

where $S_{n n}$ is the refection coefficient from the port $P_{n}$ which is attached to the $n$-th resonator, and $S_{m n}$ is the transmission coefficient from port $P_{n}$ to $P_{m}$. In the real frequency domain, the external quality factors and the coupling matrix can be found by

$$
Q_{e, i}=q_{e, i} / F B W, M_{i j}=m_{i j} \cdot F B W
$$

To generate the coupling matrix, a synthesis method based on optimization [18] is applied. For the optimization to converge fast, a carefully chosen initial matrix is highly desired. In this case, two $5^{\text {th }}$-order filters, each with one transmission zero, have been first looked into and their coupling matrices are obtained to provide the performance corresponding to each channel with the prescribed channel bandwidth, return loss (20 $\mathrm{dB}$ ) and transmission zero. Then the initial matrix of the diplexer is formed by combining the two matrices from the individual filters. After optimization, the coupling coefficients and the external quality factors can be obtained as follows.

Mutual couplings: $m_{1,2}=0.1345, m_{2,3}=0.0537, m_{3,4}=$ $0.0495, m_{4,5}=0.0837 ; m_{1,6}=0.1499, m_{6,7}=0.0667, m_{7,8}$ $=0.0653, m_{8,9}=0.0109 ; m_{2,4}=-0.0398, m_{6,8}=0.048$;

Self-couplings: $m_{1,1}=0, m_{2,2}=-0.1385, m_{3,3}=-0.0778$, $m_{4,4}=-0.1479, m_{5,5}=-0.1457, m_{6,6}=0.1156, m_{7,7}=$ $0.0377, m_{8,8}=0.1218, m_{9,9}=0.119$;
External quality factors: $q_{e, 1}=4.0797, q_{e, 5}=10.1085$, $q_{e, 9}=7.5955$.

It can be seen from the coupling matrix that only the common resonator is tuned to $f_{0}\left(m_{1,1}=0\right)$ whereas the others are asynchronously tuned $\left(m_{i, i} \neq 0\right.$ when $\left.i \neq 1\right)$. On the other hand, $m_{2,4}$ and $m_{6,8}$ are non-zero and opposite in signs because of the requirement of asymmetric transmission zeros. The responses calculated using (4), (5) and the acquired coupling matrix are shown in Fig. 2. It can be seen that $30 \mathrm{~dB}$ isolation between the two channels is achieved due to the introduction of the asymmetric transmission zeros.

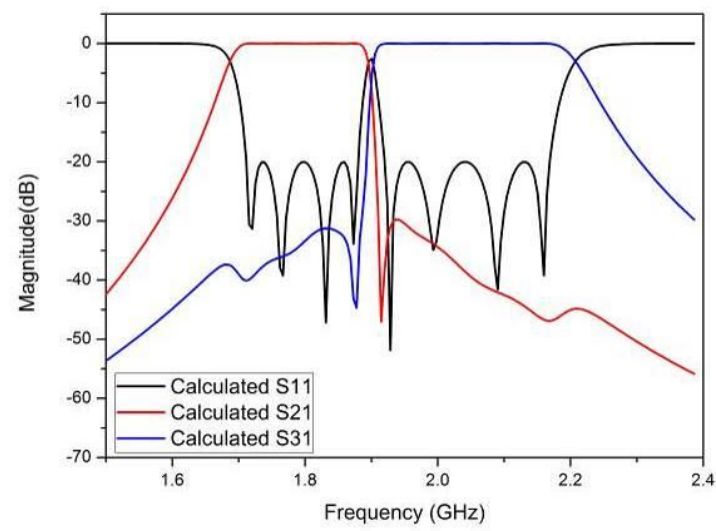

Fig. 2. Calculated responses of the diplexer from the acquired coupling matrix

\section{B. Simulation}

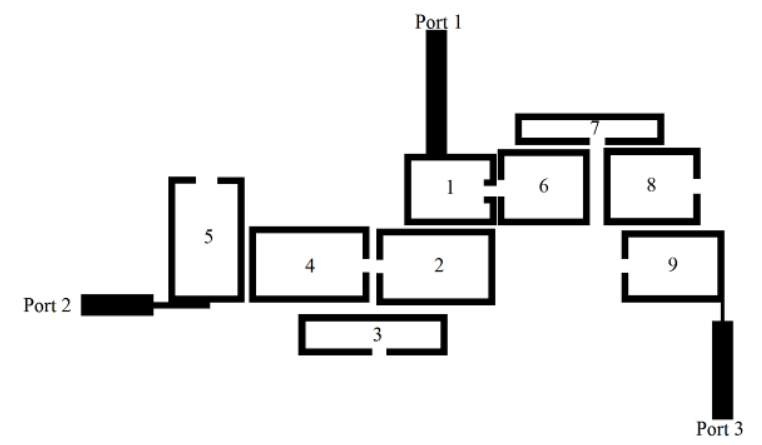

Fig. 3. Layout of the diplexer. The width of the feed lines is set to be $3.1 \mathrm{~mm}$ The gaps $d_{i k}$ between the resonators are $d_{12}=0.56 \mathrm{~mm}, d_{23}=1.46 \mathrm{~mm}, d_{34}=$ $1.84 \mathrm{~mm}, d_{45}=0.74 \mathrm{~mm}, d_{24}=1.16 \mathrm{~mm} ; d_{16}=0.22 \mathrm{~mm}, d_{67}=0.74 \mathrm{~mm}, d_{78}=$ $0.56 \mathrm{~mm}, d_{89}=0.88 \mathrm{~mm}, d_{68}=2.14 \mathrm{~mm}$.

A Rogers RO4003C substrate with a dielectric constant of 3.55 , a loss tangent of 0.0029 (nominal value at $10 \mathrm{GHz}$ ) and a thickness of $1.524 \mathrm{~mm}$ is selected for the design. Open loop resonators with a line width of $1 \mathrm{~mm}$ are chosen for the construction of the diplexer because they not only accommodate a compact layout, but also allow both positive and negative cross-couplings to be realized [16, 19]. To extract the coupling values from the simulated S-parameters, firstly the resonance frequency of each asynchronously tuned resonator is found by using the expression [20] 


$$
f_{i}=\frac{f_{0}}{\sqrt{1-m_{i i}}}
$$

where $i$ denotes the index of the resonator and $f_{0}$ is $1.94 \mathrm{GHz}$. The layout and of the microstrip circuit is shown in Fig. 3.

Resonator 2-3-4 and resonator 6-7-8 are designed as two trisection structures. The coupling between 2 and 4 is dominantly electric whereas the coupling between 6 and 8 is magnetic. The simulated weakly-coupled transmission responses of these trisection structures are shown in Fig. 4. It can be seen that transmission zeros are generated at the different sides due to the opposite sign of the couplings [16].

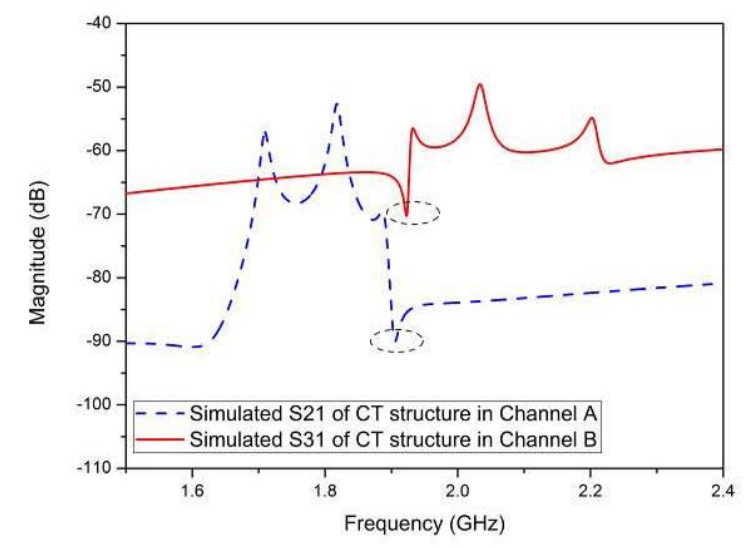

Fig. 4. Simulated transmission response of the trisection structures.

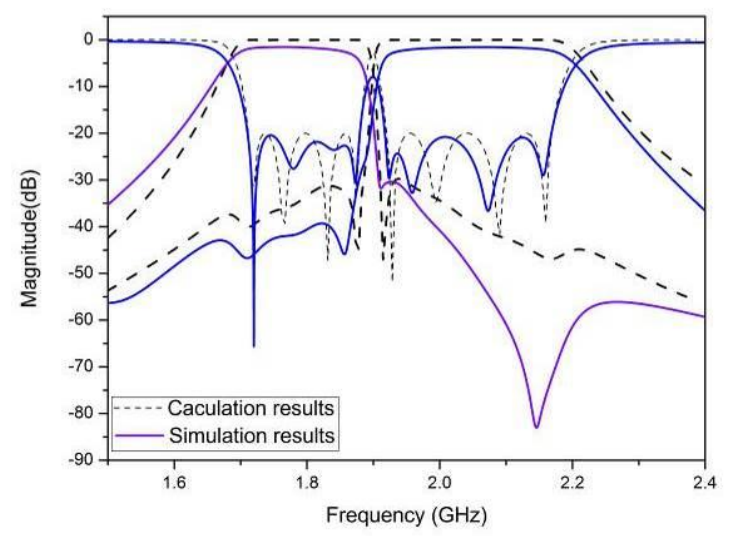

Fig. 5. Simulated results of the diplexer in comparison with the calculated.

The wide bandwidth demands strong external couplings. So the feed lines of the diplexer are tapped to the input and output resonators. External quality factors are extracted from the group delay and the phase of $S_{11}$ [17]. The simulated transmission responses as compared with the calculated ones are shown in Fig. 5. The simulated insertion loss in each channel is $1.54 \mathrm{~dB}$ and $1.56 \mathrm{~dB}$ respectively, and the return losses are better than $20 \mathrm{~dB}$ in both channels. Isolations between the two channels are $28 \mathrm{~dB}$. It can be seen that the simulated responses of channel $B$ agree well with the calculations whereas there is a small difference in channel A. The simulated lower side slope is flatter than the calculated. This is believed to be a result of the unexpected transmission zero at $2.15 \mathrm{GHz}$. This transmission zero can be attributed to the spurious cross coupling between resonator 1 and 4 which is not accounted for in the coupling matrix [21].

\section{FABRICATION AND MEASUREMENTS}

Fabrication was made by LPKF ProtoMat S63 micro milling process and the prototype is shown in Fig. 6. The whole dimension of the circuit is $110 \mathrm{~mm} \times 60 \mathrm{~mm}$ and the minimum gap used is $0.2 \mathrm{~mm}$.

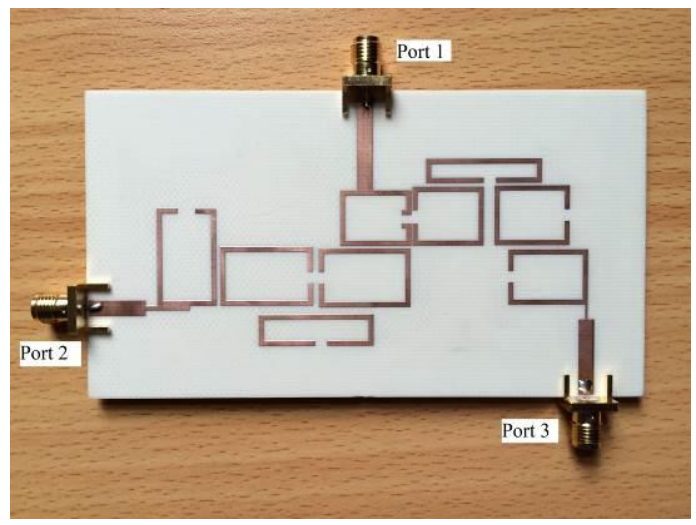

Fig. 6. Photograph of the fabricated diplexer.

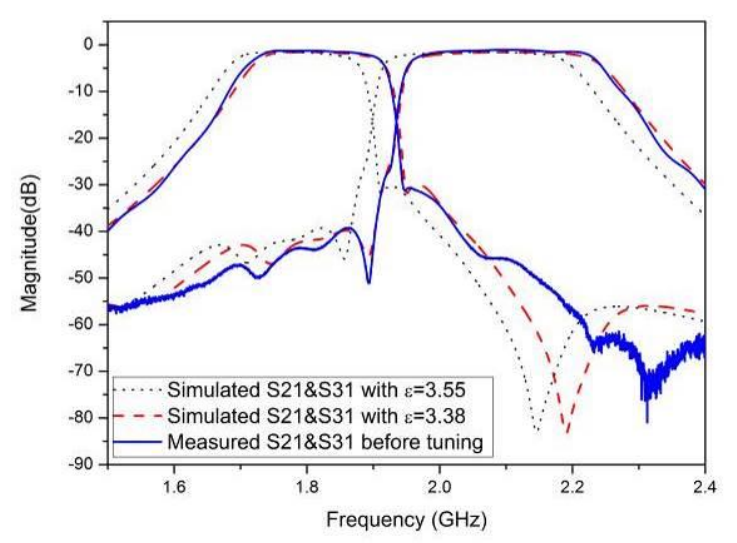

Fig. 7. Measured transmission responses of the diplexer in comparison with simulaitons.

The measurement was taken by Agilent N5230A network analyzer. Shown in Fig. 7 are the measured results as compared with two sets of simulations - one using a dielectric constants of 3.55 (the so-called design DK) and the other using 3.38 (the process DK from the supplier's date sheets). It can been seen that the measured frequency responses are higher than the simulated results with the dielectric constant 3.55 , but almost in perfect agreement with the results from the dielectric constant 3.38. The apparent discrepancy from the use of the design DK may be caused by the tight edge couplings in the circuit as required by the broad overall bandwidth of the diplexer. Finally, 
the measured insertion losses are $1.31 \mathrm{~dB}$ and $1.2 \mathrm{~dB}$ in channel $\mathrm{A}$ and $\mathrm{B}$.

The measured reflection responses of the diplexer, after tuning, are shown in Fig. 8. The simulation result with the dielectric constant of 3.38 is also displayed for comparison. A return loss of $20 \mathrm{~dB}$ and $18 \mathrm{~dB}$ is achieved in channel $\mathrm{A}$ and channel B respectively. It can also be found there are four reflection zeros in each channel as expected from the simulation. However, the post-fabrication tuning increases the bandwidth of channel A. The overall response shifts to lower frequencies because of the loading effect of the tuning materials.

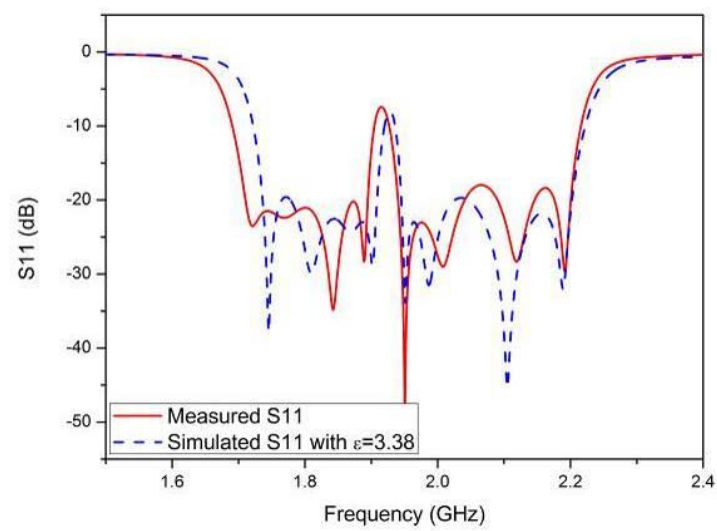

Fig. 8. Measured refection responses of the diplexer after tuning.

\section{CONCLUSION}

This paper presents a wideband microstrip diplexer with adjacent channels. The design is based on an all-resonator structure, and the overall bandwidth is $23.7 \%$. Two trisection structures are used in order to make asymmetric transmission zeros to increase the isolation between the two channels. The diplexer is designed by using open loop resonators. The measured transmission response results show an insertion loss of $1.31 \mathrm{~dB}$ and $1.2 \mathrm{~dB}$ in channel $\mathrm{A}$ and channel $\mathrm{B}$ respectively, and an isolation of $28 \mathrm{~dB}$ between the two channels. Reasonably good agreement has been achieved between simulations and measurements.

\section{REFERENCES}

[1] R. V. Snyder, A. Mortazawi, I. Hunter, S. Bastioli, G. Macchiarella, and $\mathrm{K}$. Wu, "Present and Future Trends in Filters and Multiplexers," IEEE Trans. Microw. Theory Tech., vol. 63, pp. 3324-3360, 2015.
[2] F. Boone and W. Ke, "Nonradiative dielectric (NRD) waveguide diplexer for millimeter-wave applications," in IEEE MTT-S Int. Microw. Symp. Digest, 2003, pp. 1471-1474 vol.3.

[3] J. An, G. M. Wang, C. X. Zhang, and P. Zhang, "Diplexer using composite right-/left-handed transmission line," Electron. Lett., vol. 44, pp. 685-687, 2008.

[4] T. Yang, P. L. Chi, and T. Itoh, "High Isolation and Compact Diplexer Using the Hybrid Resonators," IEEE Microw. Wireless Compon. Lett., vol. 20, pp. 551-553, 2010.

[5] M. L. Chuang and M.-T. Wu, "Microstrip Diplexer Design Using Common T-Shaped Resonator," IEEE Microw. Wireless Compon. Lett., vol. 21, pp. 583-585, 2011.

[6] P. Han-Sam and C. Yi-Chyun, "Microstrip Diplexer Constructed With New Types of Dual-Mode Ring Filters," IEEE Microw. Wireless Compon. Lett., vol. 25, pp. 7-9, 2015.

[7] W.-H. Tu, W.-C. Hung, and T.-H. Du, "Design of Microwave Microstrip Multiband Diplexers for System in Package," IEEE Trans. Compon. Packag. Technol., vol. 5, pp. 502-507, 2015.

[8] C. F. Chen, T. Y. Huang, C. P. Chou, and R. B. Wu, "Microstrip diplexers design with common resonator sections for compact size, but high isolation," IEEE Trans. Microw. Theory Tech., vol. 54, pp. 1945 1952, 2006.

[9] R. J. Cameron, "General coupling matrix synthesis methods for Chebyshev filtering functions," IEEE Trans. Microw. Theory Tech., vol. 47, pp. 433-442, 1999.

[10] F. Loras-Gonzalez, S. Sobrino-Arias, I. Hidalgo-Carpintero, D. SegoviaVargas, and M. Salazar-Palma, "A novel Ku-band dielectric resonator triplexer based on generalized multiplexer theory," in IEEE MTT-S Int. Microw. Symp. Digest, 2010, pp. 884-887.

[11] G. Macchiarella and S. Tamiazzo, "Synthesis of Star-Junction Multiplexers," IEEE Trans. Microw. Theory Tech., vol. 58, pp. 37323741, 2010.

[12] M. Sagawa, M. Makimoto, and Y. Sadahiko, "A Design Method of Bandpass Filters Using Dielectric-Filled Coaxial Resonators (Short Papers)," IEEE Trans. Microw. Theory Tech., vol. 33, pp. 152-157, 1985

[13] D. Swanson and G. Macchiarella, "Microwave filter design by synthesis and optimization," IEEE Microw. Mag., vol. 8, pp. 55-69, 2007.

[14] X. B. Shang, Y. Wang, W. Xia, and M. J. Lancaster, "Novel Multiplexer Topologies Based on All-Resonator Structures," IEEE Trans. Microw. Theory Tech., vol. 61, pp. 3838-3845, 2013.

[15] Y. Wang and M. J. Lancaster, "An Investigation on the coupling characteristics of a novel multiplexer configuration," in Eur. Microw. Conf.e (EuMC), 2013 European, 2013, pp. 900-903.

[16] J. S. Hong and M. J. Lancaster, "Microstrip cross-coupled trisection bandpass filters with asymmetric frequency characteristics," Microwaves, Antennas and Propagation, IEE Proceedings, vol. 146, pp. 84-90, 1999.

[17] J. S. Hong and M. J. Lancaster, "Microstrip filters for RF/microwave applications," Wiley, 2001.

[18] X. Shang, Y. Wang, G. L. Nicholson, and M. J. Lancaster. (2012, Design of multiple-passband filters using coupling matrix optimisation. IET Microwaves Antennas Propag. 6(1), 24-30.

[19] J. S. Hong and M. J. Lancaster, "Couplings of microstrip square openloop resonators for cross-coupled planar microwave filters," IEEE Trans. Microw. Theory Tech., vol. 44, pp. 2099-2109, 1996.

[20] C. H. Lin, C. H. Wang, and C. H. Chen, "A Simple Design Procedure for the Asynchronous Box-Section Filter," in Micro. Conf., 2007. APMC 2007. Asia-Pacific, 2007, pp. 1-4.

[21] J. B. Thomas, "Cross-coupling in coaxial cavity filters - a tutorial overview," IEEE Trans. Microw. Theory Tech., vol. 51, pp. 1368-1376, 2003. 